\title{
Deviations in point of zero charge with nanoscale pore geometries of synthesized silica-based materials
}

A. T. JaCOBSON*1 ${ }^{*}$, J. C. DeweY ${ }^{1}$, M. G. MuraleEdHARAN ${ }^{2}$, C.CHEN ${ }^{2}$, A. C. T. van DUIN ${ }^{2}$, G. C. COPELAND ${ }^{1}$, J. P.

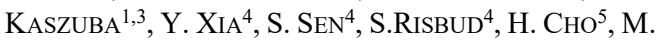

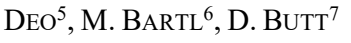

${ }^{1}$ Geology \& Geophysics, U. of Wyoming, Laramie, WY, (*correspondence: ajacobs8@uwyo.edu)

${ }^{2}$ Mechanical Engineering, Pennsylvania State, University Park, PA

${ }^{3}$ School of Energy Resources, U. of Wyoming, Laramie, WY

${ }^{4}$ Materials Science \& Engineering, UC-Davis, Davis, CA

${ }^{5}$ Chemical Engineering, U. of Utah, Salt Lake City, UT

${ }^{6}$ Chemistry, U. of Utah, Salt Lake City, UT

${ }^{7}$ Metallurgical Engineering, U. of Utah, Salt Lake City, UT

Point of zero charge (PZC) is an important surface characteristic in understanding reactions between fluids and solid surfaces. PZC describes the condition in which a surface has an electrical charge density equal to zero. In a solidaqueous system absent of any competing ions, protons and hydroxyls govern changes in surface charge of solids. PZC is thus defined by $\mathrm{pH}$ which results in a net-neutral surface charge. Due to the dependence of surface reactions and material properties on surface charge and speciation, $\mathrm{PZC}$ is an important parameter in understanding geological and commercial processes including, but not limited to, adsorption, catalysis, enhanced oil recovery, and carbon sequestration.

PZC is well documented among bulk macroporous materials, however, preliminary results of water confined in meso and micropores indicate the PZC of nanoscale porous materials deviate from their macroporous counterparts. Furthermore, results suggest different pore geometries of otherwise equivalent mesoporous materials also affect PZC. Three mesoporous $\mathrm{SiO}_{2}$ materials (MCM-41, SBA-15, and SBA-16) with similar pore sizes but different pore geometries were compared. The PZC of each material was determined using both increasing and decreasing potentiometric titrations at four ionic strengths $\left(0.001-1 \mathrm{M} \mathrm{KNO}_{3}\right)$ in a $\mathrm{pH}$ range of 2 to 10. $\mathrm{PZC}$ values are verified using electrophoresis to measure zeta potentials. ReaxFF simulations of pore geometries using metadynamics quantify the free energy barrier for surface proton dissociation, which permits calculation of $\mathrm{pKa}$ values for the dissociation reactions. PZC is then obtained from the $\mathrm{pKa}$ values for comparison to experimental results. Surface complexation models are also used to evaluate the proton affinity of surface sites. The models are an additional validation tool for experiments and simulations. 